\title{
Dynamic of a TB-HIV Coinfection Epidemic Model with Latent Age
}

\author{
Xiaoyan Wang, Junyuan Yang, and Fengqin Zhang \\ Department of Applied Mathematics, Yuncheng University, Yuncheng 044000, Shanxi, China \\ Correspondence should be addressed to Junyuan Yang; yangjunyuan00@126.com
}

Received 16 November 2012; Accepted 6 January 2013

Academic Editor: Jinde Cao

Copyright ( 2013 Xiaoyan Wang et al. This is an open access article distributed under the Creative Commons Attribution License, which permits unrestricted use, distribution, and reproduction in any medium, provided the original work is properly cited.

\begin{abstract}
A coepidemic arises when the spread of one infectious disease stimulates the spread of another infectious disease. Recently, this has happened with human immunodeficiency virus (HIV) and tuberculosis (TB). The density of individuals infected with latent tuberculosis is structured by age since latency. The host population is divided into five subclasses of susceptibles, latent TB, active TB (without HIV), HIV infectives (without TB), and coinfection class (infected by both TB and HIV). The model exhibits three boundary equilibria, namely, disease free equilibrium, TB dominated equilibrium, and HIV dominated equilibrium. We discuss the local or global stabilities of boundary equilibria. We prove the persistence of our model. Our simple model of two synergistic infectious disease epidemics illustrates the importance of including the effects of each disease on the transmission and progression of the other disease. We simulate the dynamic behaviors of our model and give medicine explanations.
\end{abstract}

\section{Introduction}

Coepidemics - the related spread of two or more infectious diseases-have afflicted mankind for centuries. Worldwide, there were an estimated 1.37 million coinfected HIV and TB patients globally in 2007. Around 80 percent of patients live in sub-Saharan Africa. 456000 people died of HIV-associated TB in 2007. HIV/AIDS and tuberculosis (TB) are commonly called the "deadly duo" and referred to as HIV/TB, despite biological differences. HIV is a retrovirus that is transmitted primarily by homosexual and heterosexual contact, needle sharing, and from mother to child. The disease eventually progresses to AIDS as the immune system weakens. HIV can be treated with highly active antiheroical therapy (HAART), but there is presently no cure [1]. Virtually all HIV-infected individuals can transmit the virus to others, and an infected individual's chance of spreading the virus generally increases as the disease progresses and damages the immune system [2]. Tuberculosis is caused by mycobacterium tuberculosis bacteria and is spread through the air. Some TB infections are "latent," meaning that a person has the TB-causing bacteria but it is dormant. A person with latent TB is not sick and is not infectious. However, latent TB can progress to "active" TB. "Active" TB infection means that the TB bacteria are multiplying and spreading in the body. A person with active $\mathrm{TB}$ in their lungs or throat can transmit the bacteria to others. Symptoms of active TB include a cough that lasts several weeks, weight loss, loss of appetite, fever, night sweats, and coughing up blood.

HIV weakens the immune system and so people are more susceptible to catching TB if they are exposed. At least onethird of people living with HIV worldwide are infected with TB and are 20-30 times more likely to develop TB than those without HIV. TB bacteria accelerate the progression of HIV to AIDS. Persons coinfected with TB and HIV may spread the disease not only to the other HIV-infected persons, but also to members of the general population who do not participate in any of the high risk behaviors associated with HIV. People living with HIV and displaying early diagnosis need treatment in time. If TB is present, they should receive TB preventive treatment (IPT). The treatments are not expensive. Therefore, it is essential that adequate attention must be paid to study the transmission dynamics of HIV-TB coinfection in the population. Current treatment for HIV is known as highly active antiretroviral therapy (HAART) [3].

Some authors have developed simulation models to investigate HIV-TB co-epidemic dynamics [4-7]. West and 


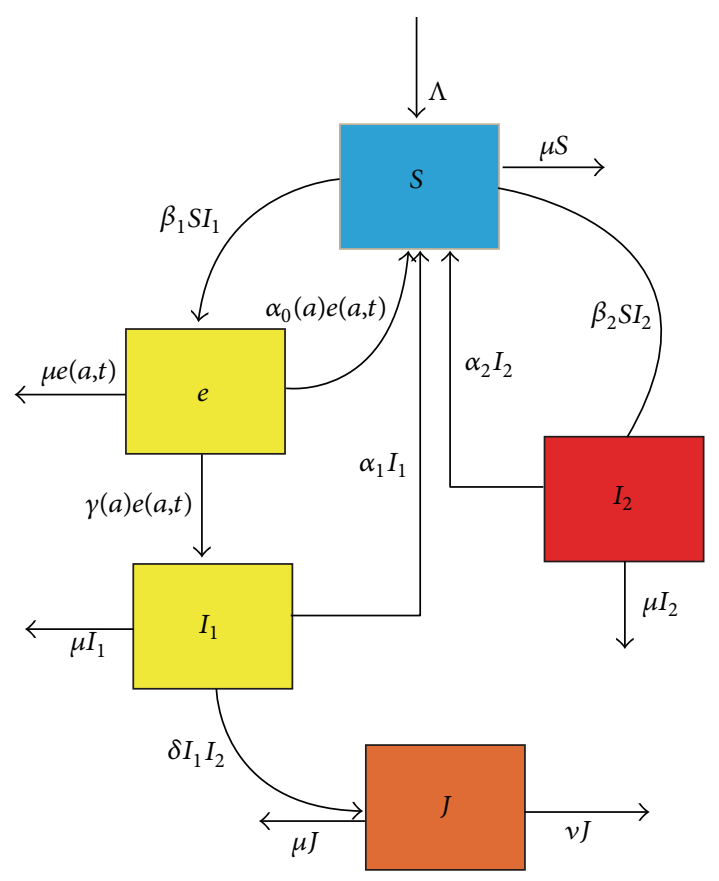

FIGURE 1: The flow diagram of the model (1). Two yellow rectangulars denote the individuals infected by latent tuberculosis and active tuberculosis. The red rectangular denotes the individuals infected by HIV. The orange rectangular denotes the individuals coinfected by TB and HIV.

Thompson [8] developed models which reflect the transmission dynamics of both TB and HIV and discussed the magnitude and duration of the effect that the HIV epidemic may have on TB. Naresh and Tripathi [9] presented a model for HIV-TB coinfection with constant recruitment of susceptibles and found that TB will be eradicated from the population if more than 90 percent TB infectives are recovered due to effective treatment. Naresh et al. [10] studied the effect of tuberculosis on the spread of HIV infection in a logistically growing human population. They found that as the number of $\mathrm{TB}$ infectives decreases due to recovery, the number of HIV infectives also decreases and endemic equilibrium tends to TB free equilibrium. Long et al. [11] discussed two synergistic infectious disease epidemics illustrating the importance of including the effects of each disease on the transmission and progression of the other disease.

However, these models may exclude coinfection, may greatly simplify infection dynamics, and may include few disease states (e.g., the important distinction between latent and active TB was absent). They assume that HIV treatment is unavailable. Bifurcation will appear in some models $[12,13]$. We perform the additional latent age, analyze the coexistence equilibria, and extend the model to include disease recovery in this paper. At the same time, we obtain the persistence of the system and global stabilities of the equilibria under some conditions.

This paper is organized as follows. In Section 2, we introduce the TB-HIV coinfection model. In Section 3, we introduce the reproduction numbers of TB and HIV $R_{1}, R_{2}$ and discuss the existence of the equilibria. The values of the disease-free equilibrium, the two boundary equilibria, and the coexistence equilibrium are given explicitly. Section 4 focuses on local and global stabilities of the equilibria. In Section 5, we discuss the persistence of the system in suitable period. In Section 6, we simulate and illustrate our results. We give some biological explanations in this section. In Section 7, we conclude our results and discuss the defect of our model.

\section{The Model Formulation}

Two diseases mentioned in the introduction are spreading in a population of total size $N(t)$. We classify the total population into four classes: the susceptible $S(t)$; the individuals infected by the tuberculosis, $e(a, t)$ and $I_{1}(t)$ denotes the latent TB class which has no infectious ability and active TB class who can infect the susceptible class, respectively; the individuals infected by HIV $I_{2}(t)$; the individuals coinfected by tuberculosis and HIV $J(t)$. The individuals infected with active TB infect the susceptible and then develop into the latent individuals at $\beta_{1}$. The individuals infected by HIV infect the susceptible and become the individuals infected by $\mathrm{HIV}$ at $\beta_{2}$. An individual already infected with TB can be coinfected with HIV at $\delta$ and thus become jointly infected individuals $J(t)$.

Figure 1 presents a schematic flow diagram of the mathematical model as follows:

$$
\begin{aligned}
\frac{d S}{d t}= & \Lambda-\mu S-\beta_{1} S I_{1}-\beta_{2} S I_{2} \\
& +\int_{0}^{\infty} \alpha_{0}(a) e(a, t) d a+\alpha_{1} I_{1}+\alpha_{2} I_{2}, \\
\frac{\partial e(a, t)}{\partial a}+\frac{\partial e(a, t)}{\partial t}= & -\left(\mu+\gamma(a)+\alpha_{0}(a)\right) e(a, t), \\
e(0, t)= & \beta_{1} S I_{1}, \\
\frac{d I_{1}}{d t}= & \int_{0}^{\infty} \gamma(a) e(a, t) d a-\mu I_{1}-\delta I_{1} I_{2}-\alpha_{1} I_{1}, \\
\frac{d I_{2}}{d t}= & \beta_{2} S I_{2}-\left(\mu+\alpha_{2}\right) I_{2}, \\
\frac{d J}{d t}= & \delta I_{1} I_{2}-(\mu+v) J,
\end{aligned}
$$

where $\mu$ is natural death rate and $\Lambda$ is birth rate. $\gamma(a)$ is rate of endogenous reactivation of latent TB. We assume that the individuals separately infected by TB and HIV are not lethal but the coinfection can lead to the extra death at $v$. Specifically, we assume that jointly infected individuals do not recover. Individuals infected with latent $\mathrm{TB}$, active $\mathrm{TB}$, or HIV alone may be potentially treated at rates $\alpha_{0}(a), \alpha_{1}$, and $\alpha_{2}$, respectively.

Assumption 1. Suppose that

(a) $\Lambda, \mu, \delta, \alpha_{1}, \alpha_{2}, \beta_{1}, \beta_{2} \in(0,+\infty)$; 
(b) $v \in[0,+\infty)$;

(c) $\gamma(a), \alpha_{0}(a) \in C_{B, U}([0,+\infty), R) \cap C_{+}([0,+\infty), R)$ and for each $a \geq 0$, where $C_{B U}(R)$ denotes the space of bounded and uniformly continuous map from $[0,+\infty)$ into $R$.

We assume (1) with the initial conditions:

$$
\begin{gathered}
S(0)=S_{0} \geq 0, \quad e(a, 0)=e_{0}(a) \in L_{+}^{1}(0,+\infty), \\
I_{1}(0)=I_{10} \geq 0, \quad I_{2}(0)=I_{20} \geq 0, \\
J(0)=J_{0} \geq 0 .
\end{gathered}
$$

Set

$$
\begin{gathered}
X=R \times Y \times R^{2}, \quad X_{+}=R_{+} \times Y_{+} \times R_{2}^{+}, \\
X_{0}=R \times Y_{0} \times R^{2}, \quad X_{0+}=X_{0} \cap X_{+} .
\end{gathered}
$$

with

$$
\begin{gathered}
Y=R \times L^{1}(0,+\infty), \quad Y_{+}=[0,+\infty) \times L_{+}^{1}(0,+\infty), \\
Y_{0}=\{0\} \times L^{1}(0,+\infty) .
\end{gathered}
$$

Let $D(\mathscr{A})=R \times Z \times R^{2}$, with $Z=\left\{0_{R}\right\} \times W^{1,1}(0, \infty)$, for each $x=\left(S, 0_{R}, e, I_{1}, I_{2}\right) \in X_{0}$. Define $\mathscr{A}: D(\mathscr{A}) \subset X_{0} \subset$ $X \rightarrow X$ and $\mathscr{F}: X_{0} \rightarrow X$ by

$\mathscr{A} x$

$$
=\left(\begin{array}{c}
-\mu S \\
-e(0) \\
-\frac{d e(\cdot)}{d a}-\left(\mu+\alpha_{0}(\cdot)+\gamma(\cdot)\right) e(\cdot) \\
-\mu I_{1}-\alpha_{1} I_{1} \\
-\mu I_{2}-\alpha_{2} I_{2} \\
-(\mu+\nu) J
\end{array}\right) \quad \text { if } x \in D(\mathscr{A})
$$

$\mathscr{F}(x)$

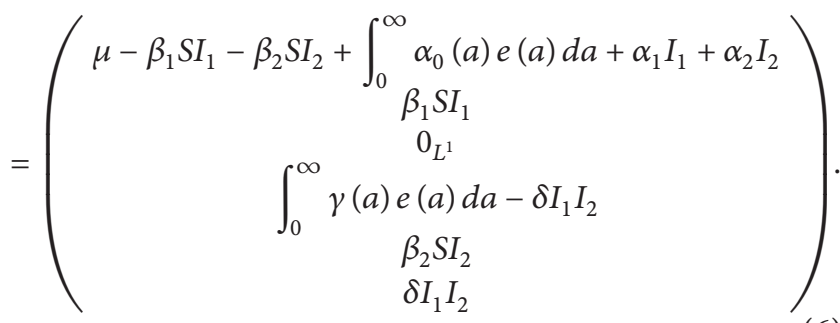

Rewrite problem (1) as an abstract Cauchy problem

$$
\frac{d u_{x}(t)}{d t}=\mathscr{A} u_{x}(t)+\mathscr{F}\left(u_{x}(t)\right), \quad t \geq 0, u_{x}(0)=x \in X_{0+} .
$$

It is well known that $\mathscr{A}$ is a Hille-Yosida operator. More precisely, we have $(-\mu,+\infty) \subset \rho(\mathscr{A})$ and for each $\lambda>-\mu$,

$$
\left\|(\lambda-\mathscr{A})^{-1}\right\| \leq \frac{1}{\lambda+\mu} .
$$

By applying the results in Magal et al. [14-16], we obtain the following proposition.

Lemma 1. There exists a uniquely determined semiflow $\{U(t)\}_{t \geq 0}$ on $X_{0+}$, such that for each $x=\left(S_{0}, 0, e_{0}, I_{10}\right.$, $\left.I_{20}, J_{0}\right)^{T} \in X_{0+}$, there exists a unique continuous map $U \in$ $C\left([0,+\infty), X_{0+}\right)$ which is an integrated solution of the Cauchy problem (1), that is to say that

$$
\begin{gathered}
\int_{0}^{t} U(s) x d s \in D(\mathscr{A}), \quad \forall t \geq 0, \\
U(t) x=x+\mathscr{A} \int_{0}^{t} U(s) x d s+\int_{0}^{t} \mathscr{F}(U(s) x) d s, \quad \forall t \geq 0 .
\end{gathered}
$$

The total population size $N(t)$ is the sum of all individuals in all classes

$$
N=S(t)+\int_{0}^{+\infty} e(a, t) d a+I_{1}(t)+I_{2}(t)+J(t) .
$$

The total population size satisfies the equation $N^{\prime}(t)=\Lambda-$ $\mu N-v J$. We introduce the notation

$$
\pi(a)=\exp \left(-\int_{0}^{a}\left(\alpha_{0}(\tau)+\gamma(\tau)\right) d \tau\right),
$$

To understand the biological meaning of the quantity $\pi(a)$ we note that $\pi(a) e^{-\mu a}$ is the probability to remain infected with TB time units after infection. In addition, we define the quantity

$$
B=\int_{0}^{+\infty} \alpha_{0}(a) \pi(a) e^{-\mu a} d a,
$$

which gives the probability of treatment since the individuals can leave TB infectious period via treatment.

$$
C=\int_{0}^{+\infty} \gamma(a) \pi(a) e^{-\mu a} d a,
$$

which gives the probability of progression since the individuals can leave TB infectious period via progression. Since individuals can only leave the latent TB infected class through treatment, progression, or death, the sum of the probabilities of recovery, progression, and death equals one, that is,

$$
\begin{aligned}
& \int_{0}^{\infty} \alpha_{0}(a) \pi(a) e^{-\mu a} d a+\int_{0}^{\infty} \gamma(a) \pi(a) e^{-\mu a} d a \\
& +\mu \int_{0}^{\infty} \pi(a) e^{-\mu a} d a=1 .
\end{aligned}
$$

It immediately follows that $B+C<1$.

\section{Equilibria of the Model with Coinfection}

We introduce the reproduction numbers of the two diseases. The reproduction number of TB is

$$
R_{1}=\frac{\Lambda \beta_{1} C}{\mu\left(\mu+\alpha_{1}\right)},
$$


and the reproduction number of HIV is

$$
R_{2}=\frac{\Lambda \beta_{2}}{\mu\left(\mu+\alpha_{2}\right)} .
$$

We note that the coinfection rate $\delta$ does not affect the reproduction numbers since coinfection does not lead to additional infections. Setting the derivatives with respect to time to zero we obtain a system of algebraic equations and one ODE for the equilibria of (1). For convenience we consider $s, e, i_{1}, i_{2}, j$ as the equilibria of the model. Therefore equilibria satisfy the following equations:

$$
\begin{gathered}
\Lambda-\beta_{1} s i_{1}-\beta_{2} s i_{2}-\mu s \\
+\int_{0}^{+\infty} \alpha_{0}(a) e(a, t) d a+\alpha_{1} i_{1}+\alpha_{2} i_{2}=0 \\
\frac{d e}{d a}=-\alpha_{0}(a) e-\mu e-\gamma(a) e \\
e(0)=\beta_{1} s i_{1}, \\
\int_{0}^{+\infty} \gamma(a) e(a) d a-\mu i_{1}-\delta i_{1} i_{2}-\alpha_{1} i_{1}=0 \\
\beta_{2} s i_{2}-\left(\mu+\alpha_{2}\right) i_{2}=0 \\
\delta i_{1} i_{2}-(\mu+v) j=0 .
\end{gathered}
$$

The ODE in the system can be solved to result in

$$
e(a)=e(0) \pi(a) e^{-\mu a}
$$

Substituting for $i$ in the integrals, one obtains

$$
\begin{gathered}
\int_{0}^{+\infty} \alpha_{0}(a) e(a) d a=e(0) \int_{0}^{+\infty} \alpha_{0}(a) \pi(a) e^{-\mu a} d a=e(0) B \\
\int_{0}^{+\infty} \gamma(a) e(a) d a=e(0) \int_{0}^{+\infty} \gamma(a) \pi(a) e^{-\mu a} d a=e(0) C
\end{gathered}
$$

With this notation the system for the equilibria becomes

$$
\begin{gathered}
\Lambda-\beta_{1} s i_{1}-\beta_{2} s i_{2}-\mu s \\
+e(0) B+\alpha_{1} i_{1}+\alpha_{2} i_{2}=0, \\
\frac{d e}{d a}=-\alpha_{0}(a) e-\mu e-\gamma(a) e, \\
e(0)=\beta_{1} s i_{1}, \\
e(0) C-\mu i_{1}-\delta i_{1} i_{2}-\alpha_{1} i_{1}=0, \\
\beta_{2} s i_{-}\left(\mu+\alpha_{2}\right) i_{2}=0, \\
\delta i_{1} i_{2}-(\mu+\nu) j=0 .
\end{gathered}
$$

This system has three boundary equilibria.

(1) The disease-free equilibrium $E_{0}=(\Lambda / \mu, 0,0,0,0)$. The disease-free equilibrium always exists.
(2) The TB dominated equilibrium exists if and only if $R_{1}>1$. The steady distribution of infectives in the $\mathrm{TB}$ equilibrium is given by

$$
\begin{gathered}
s_{1}=\frac{\Lambda}{\mu R_{1}}, \quad e=e(0) \pi(a) e^{-\mu a}, \\
i_{1}=\frac{\Lambda\left(1-1 / R_{1}\right)}{(1-B) \mu / C+(1-B-C) \alpha_{1} / C}, \\
e(0)=\beta_{1} s_{1} i_{1} .
\end{gathered}
$$

Thus, the equilibrium is

$$
E_{1}=\left(s_{1}, e, i_{1}, 0,0\right)
$$

(3) The HIV dominated equilibrium exists if and only if $R_{2}>1$ and is given by

$$
E_{2}=\left(s_{2}, 0,0, i_{2}, 0\right)
$$

where $s_{2}=1 / R_{2}, i_{2}=0, e_{2}=0, i_{2}=(\Lambda / \mu)(1-$ $\left.1 / R_{2}\right), j_{2}=0$.

(4) The coexistence equilibrium exists if and only if $R_{1}>$ $R_{2}$, and $\left[1-\left(1 / R_{2}+\mu\left(\mu+\alpha_{1}\right) / \Lambda \delta\right)\right]\left[\left(\Lambda \beta_{1} / \mu \alpha_{1} R_{2}\right)(1-\right.$ $B)-1]>0$ and it is given by

$$
E_{2}=\left(s, e, i_{1}, i_{2}, j\right)
$$

where $s=\Lambda / \mu R_{2}, e=e(0) \pi(a) e^{-\mu a}, e(0)=\beta_{1} s i_{1}, i_{1}=$ $\left(\Lambda / \alpha_{1}\right)\left(1-\left(1 / R_{2}+\mu\left(\mu+\alpha_{1}\right) / \Lambda \delta\right)\right) /\left(\left(\Lambda \beta_{1} / \mu \alpha_{1} R_{2}\right)(1-\right.$ $B)-1), i_{2}=((\mu+\delta) / \delta)\left(R_{1} / R_{2}-1\right), j=\delta i_{1} i_{2} /(\mu+\nu)$.

Notice that the values of the two dominance equilibria do not depend on the coinfection. These exact same equilibria are present even if $\delta=0$.

\section{Stability of Equilibria}

In this section we investigate local and global stabilities of equilibria. In particular, we derive conditions for the stability of the disease-free equilibrium, of the TB dominance equilibrium and of the HIV dominance equilibrium. The stability of equilibria determines whether both diseasess will be eliminated, one of the diseases will be dominated, and both diseases will persist or not.

To investigate the stability of the equilibria, we linearize the model (1). In particular, let $x(t), y(a, t), z(t), u(t)$, and $w(t)$ be the perturbations, respectively, of $s, e(a), i_{1}, i_{2}, j$. That is, $S=x+s, e=y+e, I_{1}=z+i_{1}, I_{2}=u+i_{2}, J=w+j$. Thus the perturbations satisfy a linear system. Further we consider the eigenvalue problem for the linearized system. We will denote the eigenvector again with $x, y(a), z, u$, and 
$w$. These satisfy the following linear eigenvalue problem (here $s, e, i_{1}, i_{2}$, and $j$ are the corresponding equilibria):

$$
\begin{aligned}
\lambda x= & -\beta_{1} s z-\beta_{1} i_{1} x-\beta_{2} s u-\beta_{2} i_{2} x-\mu x \\
& +\int_{0}^{\infty} \alpha_{0}(a) y(a) d a+\alpha_{1} z+\alpha_{2} u, \\
y^{\prime}(a)= & -\lambda y-\alpha_{0}(a) y-\mu y-\gamma(a) y, \\
y(0)= & \beta_{1} s z+\beta_{1} i_{1} x \\
\lambda z= & \int_{0}^{\infty} \gamma(a) y(a) d a-\mu z-\delta i_{2} z-\delta i_{1} u-\alpha_{1} z, \\
\lambda u= & \beta_{2} s u+\beta_{2} i_{2} x-\left(\mu+\alpha_{2}\right) u, \\
\lambda w= & \delta i_{2} z+\delta i_{1} u-(\mu+v) w .
\end{aligned}
$$

In the following we discuss local stability of the equilibria through the characteristic equation (25). Since the last equation has no relation with the other equations in (1) and (25), we just discuss the first four equations of them in the following.

4.1. Stability of the Disease-Free Equilibrium. For the diseasefree equilibrium we have $e(0)=i_{1}=i_{2}=j=0$, and $s=\Lambda / \mu$. Thus the system above simplifies to the following system:

$$
\begin{aligned}
\lambda x= & -\beta_{1} s z-\beta_{2} s u-\mu s \\
& +\int_{0}^{\infty} \alpha_{0}(a) y(a) d a+\alpha_{1} z+\alpha_{2} u, \\
y^{\prime}(a)= & -\lambda y-\alpha_{0}(a) y-\mu y-\gamma(a) y, \\
y(0)= & \beta_{1} s z, \\
\lambda z= & \int_{0}^{\infty} \gamma(a) y(a) d a-\mu z-\delta i_{2} z-\delta i_{1} u-\alpha_{1} z, \\
\lambda u= & \beta_{2} s u-\left(\mu+\alpha_{2}\right) u .
\end{aligned}
$$

From this system we will establish the following result regarding the local stability of the disease-free equilibrium $E_{0}$.

Theorem 2. If $R_{1}<1$ and $R_{2}<1$, then the disease-free equilibrium $E_{0}$ is locally asymptotically stable. If $R_{1}>1$ or $R_{2}>1$ then the disease-free equilibrium $E_{0}$ is unstable.

Proof. To see this, from the second to last equation we have $\lambda u=\left[\beta_{2} s-\left(\mu+\alpha_{2}\right)\right] u$, where either $\lambda=\beta_{2} s-\left(\mu+\alpha_{2}\right)$, or $u=0$. This eigenvalue $\lambda=\left(\mu+\alpha_{2}\right)\left(R_{2}-1\right)<0$, if and only if $R_{2}<1$. Thus, if $R_{2}>1$, the disease-free equilibrium $E_{0}$ is unstable because this eigenvalue is positive. Further, from the second equation we have that the remaining eigenvalues satisfying the equation,

$$
y(a)=y(0) e^{-(\lambda+\mu) a} \pi(a)=\beta_{1} s z e^{-(\lambda+\mu) a} \pi(a) .
$$

Further, from the fourth equation we have that the remaining eigenvalues satisfying the equation, also referred to as the characteristic equation, $\lambda z=\beta_{1} s \int_{0}^{\infty} \gamma(a) \pi(a) e^{-(\lambda+\mu) a} d a z-$ $\left(\mu+\alpha_{1}\right) z$. This eigenvalue $\lambda=\left(\mu+\alpha_{1}\right)\left(\beta_{1} s /\left(\mu+\alpha_{1}\right)\right)$ $\left.\int_{0}^{\infty} \gamma(a) \pi(a) e^{-(\lambda+\mu) a} d a-1\right)$, or $z=0$.

We denote the left hand side of the equation above by $\mathscr{F}_{1}(\lambda)=\lambda$, and $\mathscr{F}_{2}(\lambda)=\left(\mu+\alpha_{1}\right)\left(R_{1}(\lambda)-1\right)$, where $R_{1}(\lambda)=$ $\left(\beta_{1} s /\left(\mu+\alpha_{1}\right)\right) \int_{0}^{\infty} \gamma(a) \pi(a) e^{-(\lambda+\mu) a} d a$, and

$$
R_{1}^{\prime}(\lambda)<0, \quad \lim _{t \rightarrow+\infty} R_{1}(\lambda)=0, \quad \lim _{t \rightarrow-\infty} R_{1}(\lambda)=+\infty .
$$

If $\mathscr{F}_{1}(\lambda)=\mathscr{F}_{2}(\lambda)$ has a root with $\operatorname{Re} \lambda \geq 0$, then $\operatorname{Re} \mathscr{F}_{1}(\lambda) \geq$ 0 . But $\left|\mathscr{F}_{2}(\lambda)\right| \leq\left(\mu+\alpha_{1}\right)\left(R_{1}-1\right)<0$, when $R_{1}<1$. This is a contradiction.

Thus, if both $R_{1}<1$ and $R_{2}<1$ all eigenvalues have negative real part and the disease-free equilibrium $E_{0}$ is locally asymptotically stable. If only $R_{1}>1$ then if we consider $\mathscr{F}_{1}(\lambda)=\mathscr{F}_{2}(\lambda)$ for $\lambda$ is real, we see that $\mathscr{F}_{2}(\lambda)$ is a decreasing function of $\lambda$ approaching zero as $\lambda$ approaches infinity. Since $\mathscr{F}_{2}(0)=\left(\mu+\alpha_{1}\right)\left(R_{1}-1\right)>0$ and $\mathscr{F}_{1}(0)=0$ that implies that there is a positive eigenvalue $\lambda^{*}>0$ and the disease-free equilibrium $E_{0}$ is unstable. This concludes the proof.

In what follows, we show that the diseases vanish if $R_{1}<$ $1, R_{2}<1$.

Theorem 3. If $R_{i}<1, i=1,2$, then $E_{0}$ is a global attractor, that is, $\lim _{t \rightarrow \infty} e(a, t)=0, I_{1} \rightarrow 0, I_{2} \rightarrow 0, J \rightarrow 0$ as $t \rightarrow \infty$.

Proof. Since $N^{\prime}(t)=\Lambda-\mu N-v J \leq \Lambda-\mu N$, then $\mathrm{N}^{\infty} \leq$ $\Lambda / \mu$. Hence $S^{\infty} \leq \Lambda / \mu$. Let $\mathscr{B}(t)=e(0, t)$. Integrating this inequality along the characteristic lines we have

$$
e(a, t)= \begin{cases}\mathscr{B}(t-a) \pi(a) e^{-\mu a}, & t<a, \\ e_{0}(a-t) \frac{\pi(a)}{\pi(a-t)} e^{-\mu t}, & t \geq a .\end{cases}
$$

Since $\mathscr{B}(t)=\beta_{1} S I_{1} \leq \beta_{1} \Lambda I_{1} / \mu$ and

$$
\begin{aligned}
I_{1}^{\prime} \leq & \beta_{1} \frac{\Lambda}{\mu} \int_{0}^{t} \gamma(a) I_{1}(t-a) \pi(a) e^{-\mu a} d a \\
& +F_{1}(t)-\mu I_{1}-\alpha_{1} I_{1},
\end{aligned}
$$

where $F_{1}(t)=\int_{t}^{\infty} \gamma(a) e_{0}(a-t)(\pi(a) / \pi(a-t)) e^{-\mu t} d a$ and $\lim _{t \rightarrow \infty} F_{1}(t)=0$, from the equation for $I_{1}$ then we have the following inequality:

$$
\begin{aligned}
I_{1} \leq & I_{1}(0) e^{-\left(\mu+\alpha_{1}\right) t} \\
& +\beta_{1} \frac{\Lambda}{\mu} \int_{0}^{t} e^{-\left(\mu+\alpha_{1}\right) \tau} \int_{0}^{t-\tau} \gamma(a) I_{1}(t-a) \pi(a) e^{-\mu a} d a d \tau \\
& +\int_{0}^{t} e^{-\left(\mu+\alpha_{1}\right) \tau} F_{1}(t-\tau) d \tau,
\end{aligned}
$$


where $\gamma(a)$ is bounded and the integral of $F_{1}(t)$ goes to zero as $t \rightarrow \infty$. Consequently, taking a limsup of both sides as $t \rightarrow \infty$ we obtain

$$
I_{1}^{\infty} \leq R_{1} I_{1}^{\infty} .
$$

Since $R_{1}<1$, this inequality can only be satisfied if $I_{1}^{\infty} \rightarrow 0$, as $t \rightarrow \infty$.

Since $\mathscr{B}(t) \leq \beta_{1}(\Lambda / \mu) I_{1}$, it is easy to obtain $\mathscr{B}(t) \rightarrow 0$, as $t \rightarrow \infty$

From the equation $I_{2}$ then we have the following inequality:

$$
I_{2}^{\prime} \leq \beta_{2} \frac{\Lambda}{\mu} I_{2}-\left(\mu+\alpha_{2}\right) I_{2}
$$

Solving the inequality we get

$$
I_{2} \leq I_{2}(0) e^{-\left(\mu+\alpha_{2}\right) t}+\beta_{2} \frac{\Lambda}{\mu} \int_{0}^{\infty} I_{2}(t-\tau) e^{-\left(\mu+\alpha_{2}\right) \tau} d \tau .
$$

Consequently, taking a limsup of both sides we obtain

$$
I_{2}^{\infty} \leq R_{2} I_{2}^{\infty}
$$

If $R_{2}<1$, then $I_{2}(t) \rightarrow 0$, as $t \rightarrow \infty$.

From the fluctuations lemma, we can choose sequence $t_{n}$ such that $S\left(t_{n}\right) \rightarrow S_{\infty}$ and $S^{\prime}\left(t_{n}\right) \rightarrow 0$ when $t_{n} \rightarrow+\infty$. It follows from the first equation of (1) that we have $S_{\infty} \geq \Lambda / \mu$. Then $\Lambda / \mu \leq S_{\infty} \leq S^{\infty} \leq \Lambda / \mu$. Hence $S \rightarrow \Lambda / \mu$ when $t \rightarrow$ $\infty$. This completes the theorem.

4.2. Stability of the TB Dominated Equilibrium. In this subsection we discuss stabilities of the equilibrium $E_{1}$ and derive conditions for domination of TB. We show that the equilibrium $E_{1}$ can lose stability, and dominance of the TB is possible in the form of sustained oscillation. In this case $i_{2}=0, j=0, s=\Lambda /\left(\mu R_{1}\right), i_{1}=\Lambda\left(1-1 / R_{1}\right) /((1-B) \mu / C+$ $\left.(1-B-C) \alpha_{1} / C\right), e(a)=e(0) \pi(a) e^{-\mu a}$.

The eigenvalue problem takes the form

$$
\begin{aligned}
\lambda x= & -\beta_{1} s z-\beta_{1} i_{1} x-\beta_{2} s u-\mu s \\
& +\int_{0}^{\infty} \alpha_{0}(a) y(a) d a+\alpha_{1} z+\alpha_{2} u, \\
y^{\prime}(a)= & -\lambda y-\alpha_{0}(a) y-\mu y-\gamma(a) y, \\
y(0)= & \beta_{1} s z+\beta_{1} i_{1} x, \\
\lambda z= & \int_{0}^{\infty} \gamma(a) y(a) d a-\mu z-\delta i_{1} u-\alpha_{1} z, \\
\lambda u= & \beta_{2} s u-\left(\mu+\alpha_{2}\right) u .
\end{aligned}
$$

From the last equation we have

$$
\lambda=\left(\mu+\alpha_{2}\right)\left(\frac{R_{2}}{R_{1}}-1\right), \quad \text { or } u=0 .
$$

Hence $R_{1}>R_{2}$ the partial characteristic root of (36) is negative. Substituting

$$
y(a)=y(0) \pi(a) e^{-(\lambda+\mu) a}, \quad y(0)=\beta_{1} s z+\beta_{1} i_{1} x
$$

in the first and fourth equations and cancelling $x, z$, we arrive at the following characteristic equation:

$$
\begin{aligned}
& \left(\lambda+\mu+\alpha_{1}-\beta_{1} s C(\lambda)\right)\left(\lambda+\mu+\beta_{1} i_{1}(1-B(\lambda))\right) \\
& -\beta_{1} i_{1} C(\lambda)\left(\alpha_{1}+\beta_{1} s(B(\lambda)-1)\right)=0 .
\end{aligned}
$$

Substituting the formula

$$
1-B(\lambda)=C(\lambda)+(\lambda+\mu) E(\lambda),
$$

where

$$
E(\lambda)=\int_{0}^{\infty} \pi(a) e^{-(\lambda+\mu) a}
$$

into (39), we obtain the equivalent characteristic equation with (39) as follows:

$$
\frac{\left(\lambda+\mu+\alpha_{1}\right)\left(1+\beta_{1} i_{1} E(\lambda)\right)+\beta_{1} i_{1} C(\lambda)}{\mu+\alpha_{1}}=\frac{C(\lambda)}{C} .
$$

It is easy to see if (42) has the roots with $\operatorname{Re} \lambda>0$, the left side mode of (42) is larger than 1 , while the right side mode of (42) is less than 1, which leads to a contradiction. Hence the characteristic roots of (42) have negative real parts, then the TB dominated equilibrium $E_{1}$ is locally asymptotically stable.

Therefore we are ready to establish the first result.

Theorem 4. Let $R_{1}>1$ and $R_{1}>R_{2}$. Then, the TB dominated equilibrium $E_{1}$ is locally asymptotically stable.

For all $x=\left(S, 0_{R}, e, I_{1}, I_{2}, J\right) \in X_{0}$, we define $P_{S}: X_{0} \rightarrow$ $R, P_{I_{1}}, P_{I_{2}}: X_{0} \rightarrow R$ and $P_{I_{12}}: X_{0} \rightarrow R$ by

$$
P_{S}(x)=S, \quad P_{I_{1}}(x)=I_{1}, \quad P_{I_{2}}(x)=I_{2} .
$$

Set

$$
\begin{gathered}
M_{I_{12}}=X_{0+}, \quad M_{I_{120}}=\left\{x \in M_{I_{12}} \mid P_{I_{1}} x \neq 0, P_{I_{2}} x \neq 0\right\}, \\
\partial M_{I_{120}}=\frac{M_{I_{12}}}{M_{I_{120}}}, \quad M_{I_{1}}=\partial M_{I_{120}}, \\
M_{I_{10}}=\left\{x \in M_{I_{1}} \mid P_{I_{1}} x \neq 0, P_{I_{2}} \mathrm{x}=0\right\}, \quad \partial M_{I_{10}}=\frac{M_{I_{1}}}{M_{I_{10}}} \\
M_{I_{2}}=\partial M_{I_{10}}, \quad M_{I_{20}}=\left\{x \in M_{I_{2}} \mid P_{I_{2}} x \neq 0, P_{I_{1}} x \neq 0\right\} \\
\partial M_{I_{20}}=\frac{M_{I_{2}}}{M_{I_{20}}}, \quad M_{S}=\partial M_{I_{20}}
\end{gathered}
$$

(see Figure 2).

Lemma 5. If $R_{1}>1, \lim \inf I_{1}(t) \geq \varepsilon$ in $M_{I_{20}}$.

Proof. Let $x=\left(S_{0}, 0_{R}, e, 0_{R}, 0_{R}\right) \in M_{I_{10}}$. We assume there is a $t_{0}>0$ such that $I_{1}<\varepsilon$ for all $t \geq t_{0}$. From the first equation of (1) in $M_{I_{10}}$, it is easy to get

$$
\begin{gathered}
\frac{d S}{d t} \geq \Lambda-\varepsilon \beta_{1} S-\mu S, \\
S(0)=S_{0} .
\end{gathered}
$$




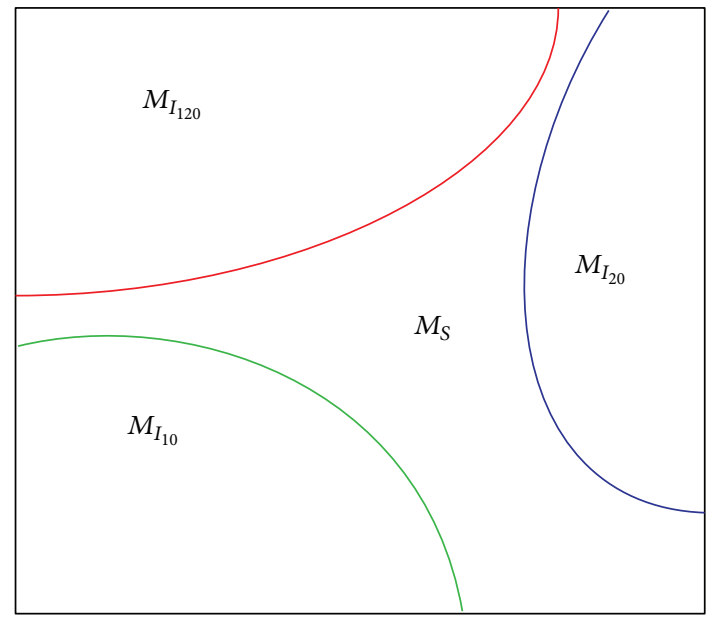

Figure 2: Zoning the area.

We solve them as follows:

$$
S(t) \geq \frac{\Lambda\left(1-e^{-\left(\mu+\beta_{1} \varepsilon\right) t}\right)}{\mu} \doteq S^{*}(t) .
$$

And $S^{*}(t) \rightarrow \Lambda / \mu$, as $t \rightarrow+\infty$. Since $R_{1}>1$, there exists $\delta>0$ and $T_{1}>0$ which satisfy $\beta_{1}(\Lambda / \mu)$ $\int_{0}^{\delta} e^{-\left(\mu+\alpha_{1}\right) s} \int_{0}^{T_{1}} \gamma(a) \pi(a) e^{-\mu a} d a d s>1$. From the second and third equations of (1), and integrating them from the characteristic line $t-a=c$, we obtain

$$
e(a, t)= \begin{cases}\beta_{1} S(t-a) I_{1}(t-a) \pi(a) e^{-\mu a}, & t \geq a, \\ e_{0}(a-t) \frac{\pi(a)}{\pi(a-t)} e^{-\mu t}, & t<a .\end{cases}
$$

From the fourth equation of (1), we get

$$
\begin{aligned}
I_{1}^{\prime}(t)= & \beta_{1} \int_{0}^{t} \gamma(a) S(t-a) I_{1}(t-a) \pi(a) e^{-\mu a} d a \\
& +\beta_{1} \int_{t}^{\infty} \gamma(a) e_{0}(a-t) \frac{\pi(a)}{\pi(a-t)} e^{-\mu t} d a \\
& -\left(\mu+\alpha_{1}\right) I_{1} .
\end{aligned}
$$

Solving it, we have

$$
\begin{aligned}
I_{1}(t) \geq & \beta_{1} \frac{\Lambda}{\mu} \int_{0}^{t} e^{-\left(\mu+\alpha_{1}\right) s} \\
& \times \int_{0}^{t-s} \gamma(a) I_{1}(t-s-a) \pi(a) e^{-\mu a} d a d s, \quad \forall t \geq t_{0} .
\end{aligned}
$$

There exist a $T_{1}>0$, such that

$$
\begin{aligned}
& I_{1}\left(t+T_{1}\right) \\
& \geq \beta_{1} \frac{\Lambda}{\mu} \int_{0}^{t+T_{1}} e^{-\left(\mu+\alpha_{1}\right) s} \\
& \quad \times \int_{0}^{t+T_{1}-s} \gamma(a) I_{1}(t-s-a) \pi(a) e^{-\mu a} d a d s, \\
& \geq \beta_{1} \frac{\Lambda}{\mu} \int_{0}^{t} e^{-\left(\mu+\alpha_{1}\right) s} \\
& \quad \times \int_{0}^{T_{1}} \gamma(a) I_{1}(t-s-a) \pi(a) e^{-\mu a} d a d s, \quad \forall t \geq t_{0} .
\end{aligned}
$$

Thus, for $t \geq \delta$, we have

$$
\begin{aligned}
& I_{1}\left(t+T_{1}\right) \\
& \geq \beta_{1} \frac{\Lambda}{\mu} \int_{0}^{\delta} e^{-\left(\mu+\alpha_{1}\right) s}
\end{aligned}
$$

$$
\times \int_{0}^{T_{1}} \gamma(a) I_{1}(t-s-a) \pi(a) e^{-\mu a} d a d s, \quad \forall t \geq t_{0} .
$$

By Assumption 1, there exists $t_{1} \geq 0$, such that $I_{1}\left(t+T_{1}\right) \geq$ 0 , for all $t+T_{1} \geq t_{1}$. Hence, there exists $\xi>0$, such that $I_{1}\left(t+T_{1}\right) \geq \xi$, for all $t+T_{1} \in\left[2 t_{1}, 2 t_{1}+\delta\right]$. Set

$$
t_{2}=\sup \left\{t+T_{1} \geq 2 t_{1}+\delta: I_{1}(l) \geq \xi, \forall l \in\left[2 t_{1}+\delta, t\right]\right\} .
$$

Assuming that $t_{2}<\infty$, it follows that

$$
\begin{aligned}
I_{1}\left(t_{2}\right) \geq & \beta_{1} \frac{\Lambda}{\mu} \int_{0}^{\delta} e^{-\left(\mu+\alpha_{1}\right) s} \\
& \times \int_{0}^{T_{1}} \gamma(a) I_{1}(t-s-a) \pi(a) e^{-\mu a} d a d s \\
\geq & \beta_{1} \frac{\Lambda}{\mu} \int_{0}^{\delta} e^{-\left(\mu+\alpha_{1}\right) s} \\
& \times \int_{0}^{T_{1}} \gamma(a) \pi(a) e^{-\mu a} d a d s \xi .
\end{aligned}
$$

Thus, $I_{1}\left(t_{2}\right)>\xi$. By the continuity for $t \rightarrow I_{1}(t)$, it follows that there exists an $\varepsilon_{1}>0$, such that $I_{1}(t) \geq \xi$, for all $t \in\left[t_{2}, t_{2}+\varepsilon_{1}\right]$ which contradicts the definition of $t_{2}$. Therefore, $I_{1}(t) \geq \xi$, for all $t>2 t_{1}$. Denote $I_{1 \infty}=$ $\liminf \operatorname{int}_{t \rightarrow+\infty} I_{1}(t) \geq \xi>0$. Using (51), it follows that $I_{1 \infty} \geq I_{1 \infty} \beta_{1}(\Lambda / \mu) \int_{0}^{\delta} e^{-\left(\mu+\alpha_{1}\right) s} \int_{0}^{T_{1}} \gamma(a) \pi(a) e^{-\mu a} d a d s$, which is impossible. Thus, $I_{1}(t) \geq \varepsilon$.

Theorem 6. If $R_{1}>1$, system (1) is permanent in $M_{I_{10}}$. Moreover there exists $A_{I_{10}}$ a compact subset of $M_{I_{10}}$ which is a global attractor for $\{U(t)\}_{t \geq 0}$ in $M_{I_{10}}$.

Proof. Suppose that $x=\left(S(t), 0, e, I_{1}, 0,0\right) \in M_{I_{10}}$ be any solution of (1). From the first equation of (1), we have

$$
S^{\prime}(t)>\mu-\left(\beta_{1}+\mu\right) S(t) \text {. }
$$


Consider the comparison equation.

$$
\begin{aligned}
& u^{\prime}(t)=\mu-\left(\beta_{1}+\mu\right) u(t), \\
& u(0)=S(0) .
\end{aligned}
$$

Similarly, (55) exists as a positive unique steady state. So we have $S(t) \geq u^{*}-\varepsilon_{1} \doteq m_{1}$, for $t$ large enough, where $u^{*}=$ $\mu /\left(\mu+\beta_{1}\right)$. From the second and third equations of (1) and Volterra's formulation, we have $e(a, t) \geq \beta_{1} m_{1} \pi(a) e^{-\mu a} \doteq m_{2}$, for $t$ large enough.

4.3. Stability of the HIV Dominated Equilibrium. In this subsection we establish that the equilibrium $E_{2}$ is locally stable whenever it exists. In this case $e(0)=0, i_{1}=0, j=0, s=$ $\Lambda / \mu R_{2}, i_{2}=(\Lambda / \mu)\left(1-1 / R_{2}\right)$. The linear eigenvalue problem becomes

$$
\begin{aligned}
\lambda x= & -\beta_{1} s z-\beta_{2} s u-\beta_{2} i_{2} x-\mu x \\
& +\int_{0}^{\infty} \alpha_{0}(a) y(a) d a+\alpha_{1} z+\alpha_{2} u, \\
y^{\prime}(a)= & -\lambda y-\alpha_{0}(a) y-\mu y-\gamma(a) y, \\
y(0)= & \beta_{1} s z, \\
\lambda z= & \int_{0}^{+\infty} \gamma(a) y(a) d a-\mu z-\delta i_{2} z-\alpha_{1} z, \\
\lambda u= & \beta_{2} s u+\beta_{2} i_{2} x-\left(\mu+\alpha_{2}\right) u .
\end{aligned}
$$

From the equation for $y(a)$ we have

$$
y(a)=y(0) \pi(a) e^{-(\lambda+\mu) a} .
$$

Substituting $y(a)$ in the equation for the initial condition $y(0)$ and assuming that $y(0) \neq 0$ we obtain the following characteristic equation:

$$
\begin{aligned}
\lambda+\delta i_{2} & =\beta_{1} s \int_{0}^{\infty} \gamma(a) \pi(a) e^{-(\lambda+\mu) a} d a-\left(\mu+\alpha_{1}\right) \\
& =\left(\mu+\alpha_{1}\right)\left(\frac{R_{1}(\lambda)}{R_{2}}-1\right) .
\end{aligned}
$$

Denoting the left hand side of the equation above by $\mathscr{F}_{1}(\lambda)=$ $\lambda+\delta i_{2}$, and $\mathscr{F}_{2}(\lambda)=\left(\mu+\alpha_{1}\right)\left(\left(R_{1}(\lambda) / R_{2}\right)-1\right)$, and also noting that

$$
R_{1}^{\prime}(\lambda)<0, \quad \lim _{t \rightarrow+\infty} R_{1}(\lambda)=0, \quad \lim _{t \rightarrow-\infty} R_{1}(\lambda)=+\infty,
$$

it is easy to see that $\mathscr{F}_{1}(\lambda)$ is an increasing function with $\lambda$. Note that $\mathscr{F}_{1}(0)=\delta I_{2} \geq 0$. But $\mathscr{F}_{2}(0) \leq\left(\mu+\alpha_{1}\right)\left(\left(R_{1} / R_{2}\right)-\right.$ $1)<0$, when $R_{1}<R_{2}$. Therefore the characteristic roots of this equation have only negative real part. Furthermore, for $y(0)=y(a)=0$, and $z=0$,

$$
\begin{aligned}
& \lambda x=-\beta_{2} s u-\beta_{2} i_{2} x-\mu x+\alpha_{2} u, \\
& \lambda u=\beta_{2} s u+\beta_{2} i_{2} x-\left(\mu+\alpha_{2}\right) u .
\end{aligned}
$$

We express $x$ from the first equation

$$
x=\frac{\left(-\beta_{2} s+\alpha_{2}\right) u}{\lambda+\mu+\beta_{2} i_{2}},
$$

and substitute it into the second equation. In addition, assuming that $u$ is nonzero, we cancel it and obtain the following characteristic equation:

$$
\lambda+\mu+\alpha_{2}=\beta_{2} s+\frac{\beta_{2} i_{2}\left(-\beta_{2} s+\alpha_{2}\right)}{\lambda+\mu+\beta_{2} i_{2}},
$$

that is,

$$
(\lambda+\mu)\left(\lambda+\mu+\alpha_{2}+\beta_{2} i_{2}-\beta_{2} s\right)=0
$$

Noticing that $\beta_{2} s=\mu+\alpha_{2}$, we obtain the following eigenvalues: $\lambda=-\mu$ and $\lambda=-\beta_{2} i_{2}$, which are both negative. Consequently, the equilibrium $E_{2}$ is locally asymptotically stable.

Therefore, we have the following theorem for equilibrium $E_{2}$.

Theorem 7. Let $R_{2}>1$. Assume that tuberculosis cannot invade the equilibrium of $H I V$, that is, $R_{1}<R_{2}$. Then the equilibrium $E_{2}$ is locally asymptotically stable.

Lemma 8. If $R_{2}>1$, then $\lim \inf I_{2}(t) \geq \varepsilon$ in $M_{I_{20}}$.

Proof. Let $x=\left(S_{0}, 0_{R}, 0_{L}^{1}, 0_{R}, I_{2}, 0_{R}\right) \in M_{I_{20}}$. We assume there is a $t_{0}>0$ such that $I_{2}<\varepsilon$ for all $t \geq t_{0}$. From the first equation of (1) in $M_{I_{20}}$, it is easy to get

$$
\begin{gathered}
\frac{d S}{d t} \geq \Lambda-\varepsilon \beta_{2} S-\mu S, \\
S(0)=S_{0} .
\end{gathered}
$$

We solve them,

$$
S(t) \geq \frac{\Lambda\left(1-e^{-\left(\mu+\beta_{2} \varepsilon\right) t}\right)}{\mu} \doteq S^{*}(t) .
$$

Then $S(t) \geq S^{*}(t)$, and $S(t) \rightarrow \Lambda / \mu$, as $t \rightarrow+\infty$. From the fourth of equation (1), $\exists t_{0}>0$, we have

$$
I_{2}^{\prime}(t) \geq \beta_{2} \frac{\Lambda}{\mu} I_{2}(t)-\left(\mu+\alpha_{2}\right) I_{2}(t), \quad \forall t \geq t_{0} .
$$

Solving it, we have

$$
I_{2}(t) \geq \beta_{2} \frac{\Lambda}{\mu} \int_{0}^{t} e^{-\left(\mu+\alpha_{2}\right)(t-s)} I_{2}(t-s) d s .
$$

We do lim inf on both side of (67), and denote $I_{2 \infty}=$ $\liminf \operatorname{int}_{t \rightarrow+\infty} I_{2}(t)>0$, thus

$$
I_{2 \infty} \geq R_{2} I_{2 \infty}
$$

which is impossible. Thus, $I_{2}(t) \geq \varepsilon$. 
Theorem 9. If $R_{2}>1$, system (1) is permanent in $M_{I_{20}}$. Moreover there exists $A_{I_{20}}$ a compact subset of $M_{I_{20}}$ which is a global attractor for $\{U(t)\}_{t \geq 0}$ in $M_{I_{20}}$.

Proof. Suppose that $x=\left(S(t), 0,0, I_{2}(t), 0\right) \in M_{I_{20}}$ be any solution of (1). From the first equation of (1), we have

$$
S^{\prime}(t)>\Lambda-\left(\beta_{2}+\mu\right) S(t) \text {. }
$$

Consider the comparison equation

$$
\begin{gathered}
u^{\prime}(t)=\Lambda-\left(\beta_{2}+\mu\right) u(t), \\
u(0)=S(0) .
\end{gathered}
$$

Similarly, (70) exists as a positive unique solution. So we have $S(t) \geq u^{*}-\varepsilon_{1} \doteq m_{2}$, for $t$ large enough, where $u^{*}=$ $\Lambda /\left(\beta_{2}+\mu\right)$.

4.4. Stability of Coexistence Equilibrium $E_{*}$. In this subsection we establish that the equilibrium $E_{*}$ is locally stable whenever it exists. In this case $s=\Lambda / \mu R_{2}, e=$ $e(0) \pi(a) e^{-\mu a}, e(0)=\beta_{1} s i_{1}, i_{1}=\left(\Lambda / \alpha_{1}\right)\left(\left(1-\left(1 / R_{2}+\mu(\mu+\right.\right.\right.$ $\left.\left.\left.\left.\alpha_{1}\right) / \Lambda \delta\right)\right) /\left(\left(\Lambda \beta_{1} / \mu \alpha_{1} R_{2}\right)(1-B)-1\right)\right), i_{2}=((\mu+\delta) / \delta)\left(R_{1} / R_{2}-\right.$ $1), j=\delta i_{1} i_{2} /(\mu+\nu)$. The characteristic equations at the coexistence equilibrium are as follows:

$$
\begin{aligned}
& \left(\lambda+\mu+\alpha_{1}+\delta i_{2}-\beta_{1} s C(\lambda)\right) \\
& \times\left(\lambda+\beta_{1} i_{1}+\beta_{2} i_{2}+\mu-\beta_{1} i_{1} B(\lambda)+\frac{\beta_{2} i_{2} \mu}{\lambda}\right) \\
& =\left(\beta_{1} s B(\lambda)-\beta_{1} s\right)\left(\beta_{1} i_{1}-\frac{\delta \beta_{2} i_{1} i_{2}}{\lambda}\right) .
\end{aligned}
$$

Theorem 10. If $R_{1}>R_{2}$, $\left[1-\left(1 / R_{2}+\left(\mu\left(\mu+\alpha_{1}\right) /\right.\right.\right.$ $\Lambda \delta))]\left[\left(\left(\lambda \beta_{1} / \mu \alpha_{1} R_{2}\right)(1-B)\right)-1\right]>0$, and the characteristic equation (71) has only negative real parts roots, then the coexistence equilibrium $E_{*}$ is locally asymptotically stable.

\section{Persistence of the System}

In this section, we consider persistence of the system in $M_{I_{120}}$ when $v=0$. It is easy to check if the system (1) is dissipative and the dynamical system is asymptotically smooth. $M_{I_{120}}, M_{I_{10}}, M_{I_{20}}$, and $M_{s}$ are positively invariant. Define

$$
\begin{gathered}
\partial \Gamma=M_{I_{10}} \cup M_{I_{20}} \cup M_{s}, \\
\partial \Gamma=M_{I_{120}} .
\end{gathered}
$$

Assuming the boundary equilibria of (1) are globally asymptotically stable, we have

$$
\begin{aligned}
\widetilde{A}_{\partial M_{I_{120}}}:=\cup_{\left(S_{0}, e_{0}(\cdot), I_{1}, I_{2}, J\right) \in \partial \Gamma} \omega\left(\left(S_{0}, e_{0}(\cdot), I_{1}, I_{2}, J\right)\right) \\
=\left\{E_{0}, E_{1}, E_{2}\right\} .
\end{aligned}
$$

By the above conclusions, it follows that $\widetilde{A}_{\partial M_{I_{120}}}$ is isolated and has an acyclic covering $M=\left\{E_{0}, E_{1}, E_{2}\right\}$. Since the orbit of any bounded set is bounded, and from Theorem 4.2 in [17], we only need to show that $E_{0}, E_{1}, E_{2}$ are ejective in $M_{I_{120}}$ if globally stable conditions of $E_{0}, E_{1}, E_{2}$ are not satisfied. Therefore, we have the following lemmas.

Lemma 11. Let Assumption 1 be satisfied and let $E_{0}$ be globally asymptotically stable. If $R_{1}>1$, then $E_{0}$ is ejective in $M_{I_{120}}$ for $\left\{U_{I_{12}}(t)\right\}_{t \geq 0}$.

Proof. Let $\delta>0, T_{1}>0$ and $\varepsilon \in(0,1)$ satisfy

$$
\beta_{1}\left(\frac{\Lambda}{\mu}-\varepsilon\right) \int_{0}^{\delta} e^{-\left(\mu+\alpha_{1}\right) s} \int_{0}^{T_{1}} \gamma(a) \pi_{1}(a) e^{-\mu a} d a d s>1
$$

Let $x=\left(S_{0}, 0_{R}, I_{10}, I_{20}, J_{0}\right) \in M_{I_{120}}$ with $\left\|x-x_{S}\right\|<\varepsilon$. Assume that

$$
\left\|U(t) x-x_{s}\right\| \leq \varepsilon, \quad t \geq 0 .
$$

Defining $S(t)=P_{S} U(t) x$, and $I_{1}(t)=P_{I_{1}} U(t) x$, for all $t \geq 0$. from (75) it follows that

$$
S(t) \geq \frac{\Lambda}{\mu}-\varepsilon, \quad \forall t \geq 0 .
$$

From the second and third equations of (1), and integrating them from the characteristic line $t-a=c$, we obtain

$$
e(a, t) \geq \begin{cases}\beta_{1}\left(\frac{\Lambda}{\mu}-\varepsilon\right) I_{1}(t-a) \pi_{1}(a) e^{-\mu a}, & t \geq a, \\ e_{0}(a-t) \frac{\pi_{1}(a)}{\pi_{1}(a-t)} e^{-\mu t}, & t<a,\end{cases}
$$

where from the fourth equation of (1), we get

$$
\begin{aligned}
I_{1}^{\prime}(t) \geq & \beta_{1} \frac{\Lambda}{\mu} \int_{0}^{t} I_{1}(t-a) \pi_{1}(a) e^{-\mu a} d a \\
& +\beta_{1} \int_{t}^{\infty} \gamma(a) e_{0}(a-t) \frac{\pi_{1}(a)}{\pi_{1}(a-t)} e^{-\mu t} d a \\
& -\left(\mu+\alpha_{1}\right) I_{1} .
\end{aligned}
$$

Solving it, we have

$$
\begin{aligned}
I_{1}(t) \geq & \beta_{1}\left(\frac{\Lambda}{\mu}-\varepsilon\right) \int_{0}^{t} e^{-\left(\mu+\alpha_{1}\right) s} \\
& \times \int_{0}^{t-s} \gamma(a) I_{1}(t-s-a) \pi_{1}(a) e^{-\mu a} d a d s, \quad \forall t \geq 0 .
\end{aligned}
$$


There exist a $T_{1}>0$, such that

$$
\begin{aligned}
& I_{1}\left(t+T_{1}\right) \\
& \geq \beta_{1}\left(\frac{\Lambda}{\mu}-\varepsilon\right) \int_{0}^{t+T_{1}} e^{-\left(\mu+\alpha_{1}\right) s} \\
& \quad \times \int_{0}^{t+T_{1}-s} \gamma(a) I_{1}(t-s-a) \pi_{1}(a) e^{-\mu a} d a d s, \\
& \geq \beta_{1}\left(\frac{\Lambda}{\mu}-\varepsilon\right) \int_{0}^{t} e^{-\left(\mu+\alpha_{1}\right) s} \\
& \quad \times \int_{0}^{T_{1}} \gamma(a) I_{1}(t-s-a) \pi_{1}(a) e^{-\mu a} d a d s, \quad \forall t \geq 0 .
\end{aligned}
$$

Thus, for $t \geq \delta$, we have

$$
\begin{aligned}
& I_{1}\left(t+T_{1}\right) \\
& \geq \beta_{1}\left(\frac{\Lambda}{\mu}-\varepsilon\right) \int_{0}^{\delta} e^{-\left(\mu+\alpha_{1}\right) s} \\
& \quad \times \int_{0}^{T_{1}} \gamma(a) I_{1}(t-s-a) \pi_{1}(a) e^{-\mu a} d a d s, \quad \forall t \geq 0 .
\end{aligned}
$$

By Assumption 1, there exists $t_{1} \geq 0$, such that $I_{1}\left(t+T_{1}\right) \geq$ 0 , for all $t+T_{1} \geq t_{1}$. Hence, there exists $\xi>0$, such that $I_{1}\left(t+T_{1}\right) \geq \xi$, for all $t+T_{1} \in\left[2 t_{1}, 2 t_{1}+\delta\right]$. Set

$$
t_{2}=\sup \left\{t+T_{1} \geq 2 t_{1}+\delta: I(l) \geq \xi, \quad \forall l \in\left[2 t_{1}+\delta, t\right]\right\} .
$$

Assume that $t_{2}<\infty$. Then

$$
\begin{aligned}
I_{1}\left(t_{2}\right) \geq & \beta_{1}\left(\frac{\Lambda}{\mu}-\varepsilon\right) \int_{0}^{\delta} e^{-\left(\mu+\alpha_{1}\right) s} \\
& \times \int_{0}^{T_{1}} \gamma(a) I_{1}(t-s-a) \pi_{1}(a) e^{-\mu a} d a d s, \\
\geq & \beta_{1}\left(\frac{\Lambda}{\mu}-\varepsilon\right) \int_{0}^{\delta} e^{-\left(\mu+\alpha_{1}\right) s} \\
& \times \int_{0}^{T_{1}} \gamma(a) \pi_{1}(a) e^{-\mu a} d a d s \xi .
\end{aligned}
$$

Thus, $I_{1}\left(t_{2}\right)>\xi$. By the continuity for $t \rightarrow I_{1}(t)$, it follows that there exists an $\varepsilon_{2}>0$, such that $I_{1}(t) \geq \xi$, for all $t \in$ $\left[t_{2}, t_{2}+\varepsilon_{2}\right]$ which contradicts the definition of $t_{2}$. Therefore, $I_{1}(t) \geq \xi$, for all $t>2 t_{1}$. Denote $I_{1 \infty}=\liminf _{t \rightarrow+\infty} I_{1}(t) \geq$ $\xi>0$. Using (81), it follows that $I_{1 \infty} \geq I_{1 \infty} \beta_{1}(\Lambda / \mu-$ $\varepsilon) \int_{0}^{\delta} e^{-\left(\mu+\alpha_{1}\right) s} \int_{0}^{T_{1}} \gamma(a) I_{1}(t-s-a) \pi_{1}(a) e^{-\mu a} d a d s$, which is impossible.

Lemma 12. Let Assumption 1 be satisfied and let $E_{1}$ and $E_{2}$ be globally asymptotically stable. Then one has the following.

(i) If $R_{1}>1$, then $x_{I_{1}}=E_{1}$ is ejective in $M_{I_{120}}$.

(ii) If $R_{2}>1$, then $x_{I_{2}}=E_{2}$ is ejective in $M_{I_{120}}$.
Theorem 13. Let Assumption 1 be satisfied and let $E_{0}, E_{1}$, and $E_{2}$ be globally asymptotically stable. Assume $R_{1}>1, R_{2}>1$. Then there exists $\varepsilon>0$ such that for all $x \in M_{I_{120}}$,

$$
\liminf \left\|P_{I_{12}} U(t) x\right\| \geq \varepsilon .
$$

Proof. It is a consequence of Theorem 4.2 in [18] applied with $\Omega\left(\partial M_{I_{120}}\right)=\left\{E_{0}\right\} \cup\left\{E_{1}\right\} \cup\left\{E_{2}\right\}$. Using Lemma 12, the result follows.

\section{Simulation}

In this section, we use (1) to examine how the prevalence of HIV impacts on TB dynamics. We also present some numerical results on the stability of $E_{0}$ (the disease-free equilibrium), $E_{1}$ (the TB dominated equilibrium), and $E_{2}$ (the HIV dominated equilibrium). We perform a numerical analysis to exhibit the TB impact on HIV under different treatments with (1). We now give three examples to illustrate the main results mentioned in the above section.

Example 14. In (1), we set $\Lambda=2, \mu=0.6, \delta=73.13, \beta_{1}=$ $0.49, \beta_{2}=0.15, v=0.9$,

$$
\begin{aligned}
& \gamma(a)= \begin{cases}0.02 & a \geq 1.49 \\
0 & 0 \leq a \leq 1.49,\end{cases} \\
& \alpha_{0}(a)= \begin{cases}0.1 & a \geq 1.49 \\
0 & 0 \leq a \leq 1.49\end{cases}
\end{aligned}
$$

$\alpha_{1}=0.01, \alpha_{2}=0.03$. We have $R_{1}=0.07438<1$ and $R_{2}=0.79365<1$, which satisfy the conditions of Theorems 2 and 3 . $E_{0}$ should be globally asymptotically stable (see Figure 3(a)). In this case, both TB and HIV will be eliminated.

Example 15. In (1), $\Lambda=2, \mu=1, \delta=4, \beta_{1}=50.49, \beta_{2}=$ $0.15, v=0.9$,

$$
\begin{gathered}
\gamma(a)= \begin{cases}0.1 & a \geq 1.49 \\
0 & 0 \leq a \leq 1.49\end{cases} \\
\alpha_{0}(a)= \begin{cases}0.1 & a \geq 1.49 \\
0 & 0 \leq a \leq 1.49\end{cases}
\end{gathered}
$$

$\alpha_{1}=0.01, \alpha_{2}=0.03$. We have $R_{1}=34.48770>1>$ $R_{2}=0.79365$, which satisfy the conditions of Theorem $4 . E_{1}$ should be globally asymptotically stable (see Figure $3(\mathrm{~b})$ ). In this case, TB is dominated in the coinfected dynamics.

Example 16. In (1), $\Lambda=2, \mu=0.6, \delta=0.0073, \beta_{1}=0.49$, $\beta_{2}=4.15, v=0.9$,

$$
\begin{gathered}
\gamma(a)= \begin{cases}0.1 & a \geq 1.49 \\
0 & 0 \leq a \leq 1.49\end{cases} \\
\alpha_{0}(a)= \begin{cases}0.1 & a \geq 1.49 \\
0 & 0 \leq a \leq 1.49\end{cases}
\end{gathered}
$$




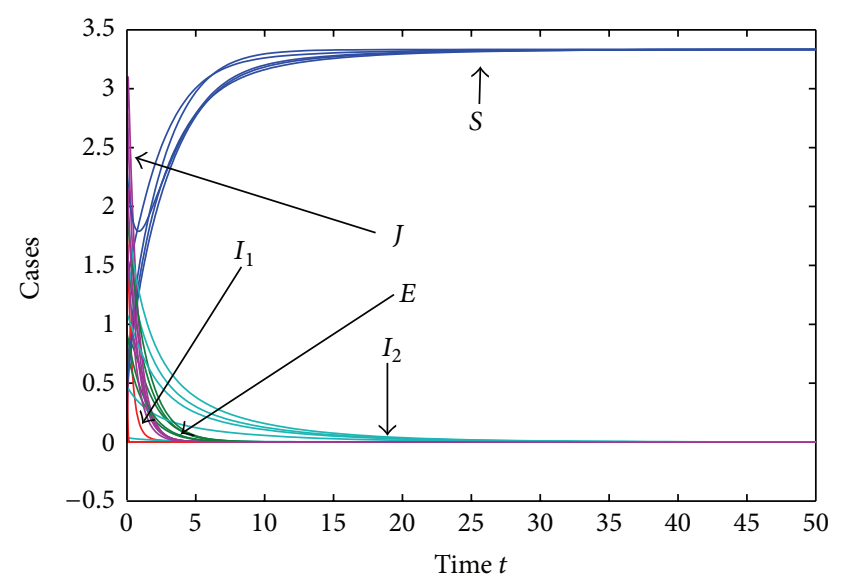

(a)

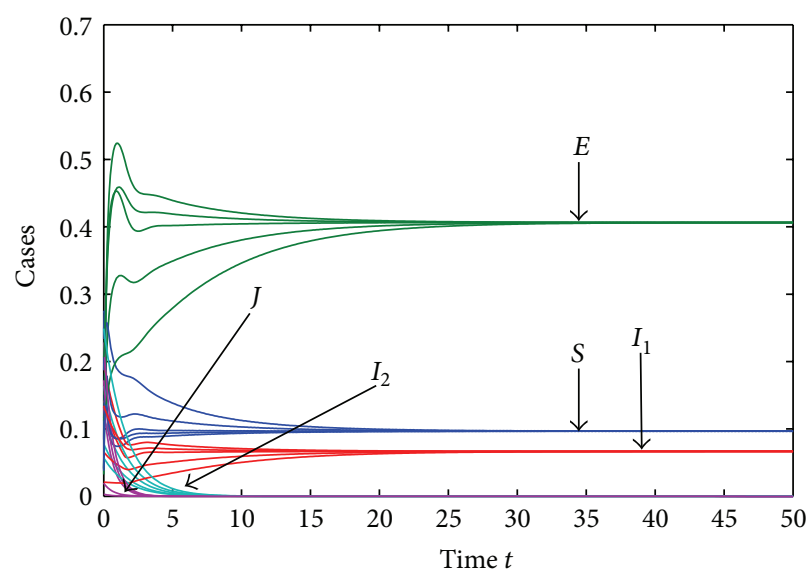

(b)

FIGURE 3: (a) show that $E_{0}$ is globally asymptotically stable. (b) shows that $E_{1}$ is globally asymptotically stable.

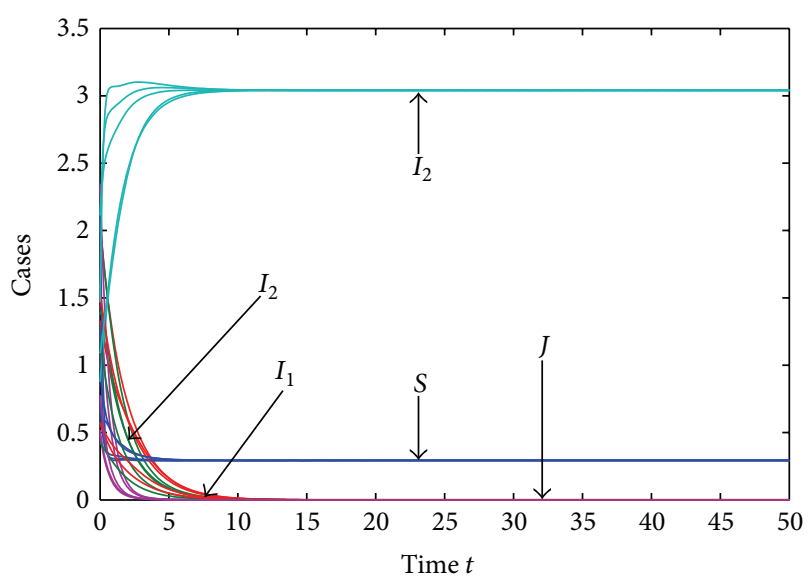

FIGURE 4: shows that $E_{2}$ is globally asymptotically stable.

$\alpha_{1}=0.01, \alpha_{2}=0.03$. We have $R_{2}=11.37566>1>$ $R_{1}=0.33470$, which satisfies the conditions of Theorem 7. $E_{2}$ should be globally asymptotically stable (see left of Figure 4 ). In this case, HIV is dominated in the coinfected dynamics.

6.1. Effect of Treatment Parameter $\alpha_{i}, \quad i=0,1,2$. We examined hypothetical treatment to gain insight into the underlying coepidemic dynamics. We considered nine treatment scenarios: no treatment $\alpha_{0}=0$ (latent TB treatment) (IPT), $\alpha_{0}=0.5$ (latent TB treatment) (IPT), $\alpha_{0}=1$ (latent TB treatment) (IPT), no treatment $\alpha_{2}=0, \alpha_{2}=0.5$ (HAART), and $\alpha_{2}=1$ (HAART), where $\alpha_{i}, i=0,1$ denotes the fraction of the eligible population receiving the corresponding treatment.

First, we considered the effect of each type of treatment in isolation (e.g., IPT for individuals with treatment for latent or active TB, but not AIDS). Exclusively treating people with latent TB reduced the number of new active TB cases, which subsequently decreased the number of new latent TB infections (Figure 5(a)). However, latent TB treatment had an adverse effect on the HIV epidemic: the number of new
HIV cases increased because individuals coinfected with HIV and latent TB lived longer (due to latent TB treatment) and thus could infect more people with HIV(see Figure 5(c)). Similarly, active TB treatment reduced the number of people with infectious $\mathrm{TB}$, which subsequently reduced the number of new latent and active TB cases (Figure 5(b)). Once again, new HIV cases increased due to longer life expectancy among those who were coinfected with HIV. Hence, the coinfected people have same effect of people infected by latent TB and active TB.

Second, we considered the effect of each type of treatment in isolation (e.g., HAART for individuals with AIDS, but no treatment for latent or active TB). The provision of HAART significantly slowed the HIV epidemic (Figure 6(c)). However, exclusively treating HIV-infected individuals with HAART adversely affects the TB epidemic (Figures 6(a) and 6(b)). Because HAART reduces AIDS-related mortality, treated individuals have a longer time to potentially infect others with TB, especially in the absence of any TB treatment. Thus, the numbers of new latent and active TB cases increased as more people were given HAART. However, the coinfected people have the same effect of people infected by HIV (see Figure 6(d)).

\section{Discussion}

We have developed a mathematical model for modelling the coepidemics, calculated the basic reproduction number, the disease-free equilibrium, the dominated equilibria (defined as a state where one disease is eradicated, while the other disease remains endemic), and got the conditions for local and global stability. We presented the sufficient conditions for the local stability of the TB dominated equilibrium in Theorem 4. We also obtained the persistence. In the case of the HIV dominated equilibrium, we presented the sufficient condition for the local stability in Theorem 7 . We also obtained the persistence of (1). We simulated and illustrated our analyzed results in Section 6. 


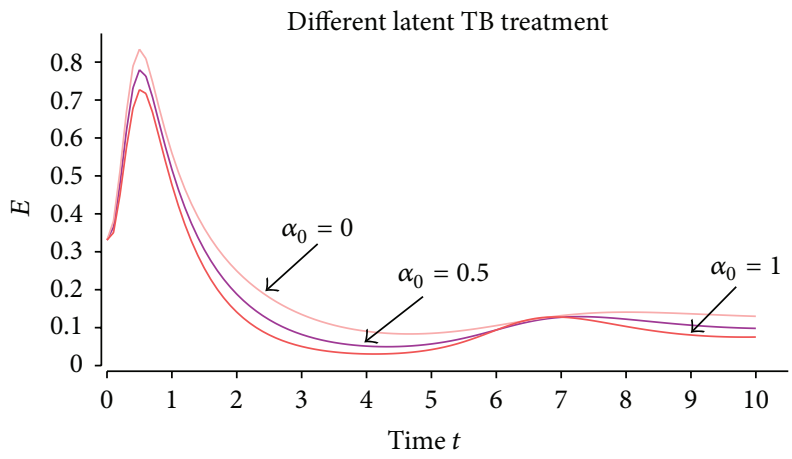

(a)

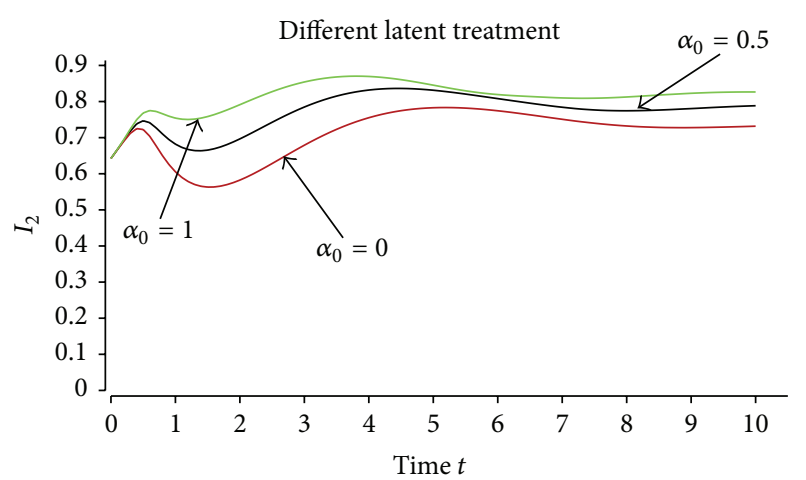

(c)

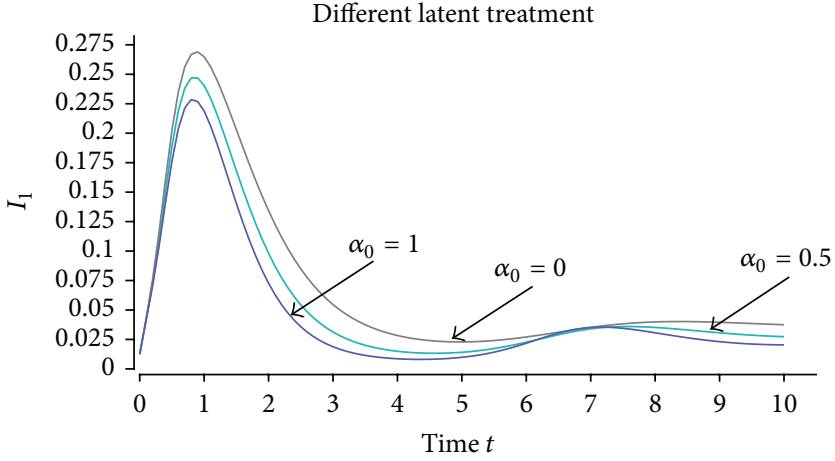

(b)

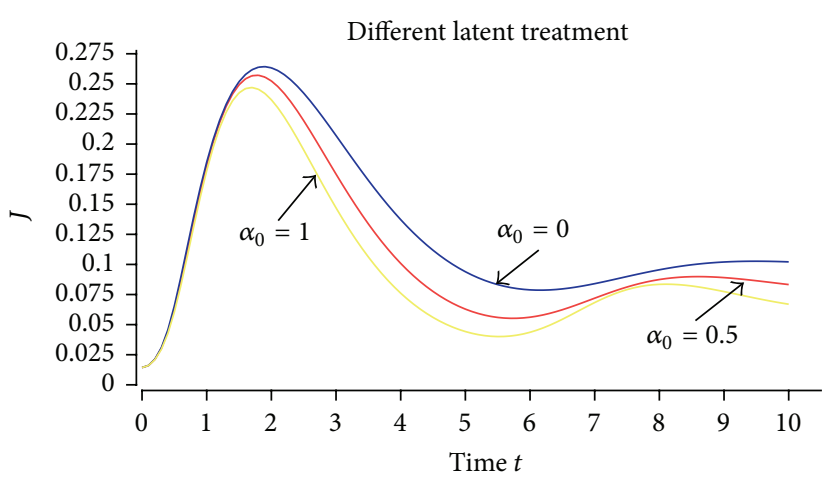

(d)

FIGURE 5: We take different latent TB treatments while we take no treatment of HAART and active TB.

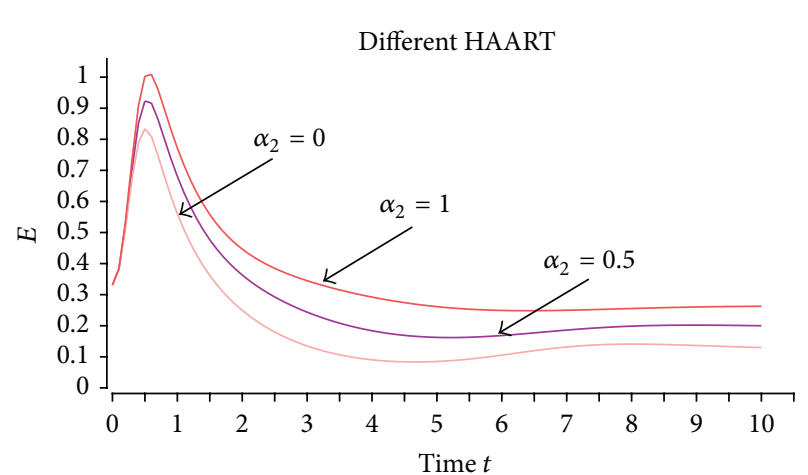

(a)

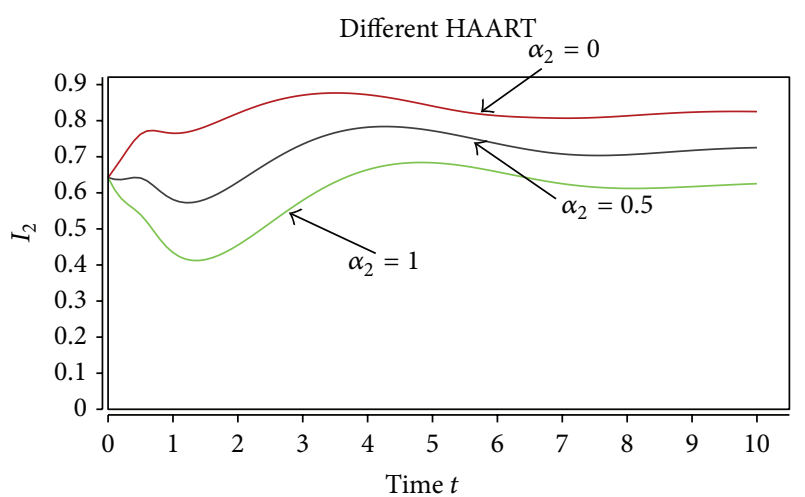

(c)

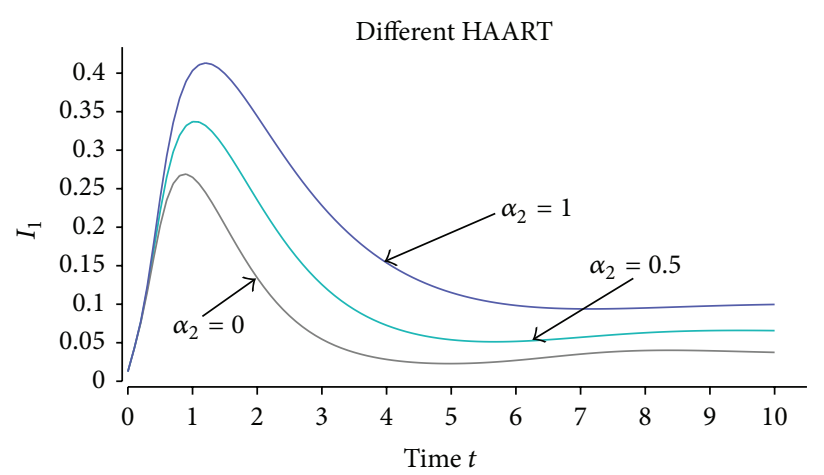

(b)

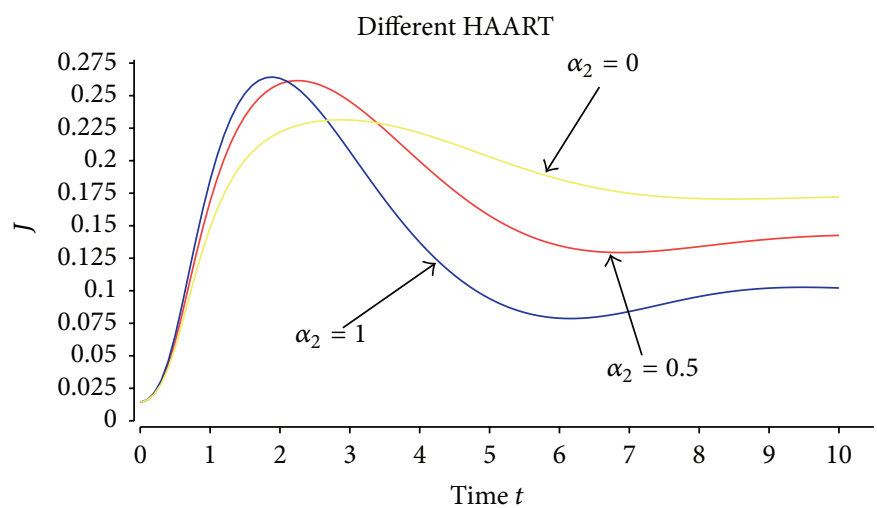

(d)

FIGURE 6: We take different HAART while we take no treatment of IPT. 
Our models have several limitations. We simplified the complicated infection dynamics of TB and HIV to develop a tractable framework, which helped us gain insights about the basic reproduction number and equilibria. We assumed uniform patterns within compartments, that is, the mixing between compartments is homogeneous. To appropriately guide policy recommendations, our coepidemic model would need to be significantly expanded. We also apply a coepidemic model to describe the HIV coinfected with other diseases, such as HIV and hepatitis C (HCV). Some $50 \%-90 \%$ of HIVinfected injection drug users are coinfected with HCV [19]. The successful HIV treatment may be adversely impacted by the presence of HCV, and HCV may cause liver damage to occur more quickly in HIV-infected individuals [19]. Modelling this kind of coinfection may play particularly important role on evaluating interventions time targeted to injection drug.

Unfortunately, we are unable yet to study the models that introduce a discrete delay and an impulsive perturbation in our model. We will explore them in the future.

\section{Acknowledgments}

This paper is supported by the NSF of China (61203228, 11071283), Mathematical Tianyuan Foundation (11226258), the Young Sciences Foundation of Shanxi (2011021001-1), and the Foundation of University (YQ-2011045, JY-2011036).

\section{References}

[1] National Institute of Allergy and Infectious Diseases (NIAID), HIV Infection and AIDS: An Overview, United States National Institutes of Health, Bethesda, Md, USA, 2006.

[2] G. D. Sanders, A. M. Bayoumi, V. Sundaram et al., "Costeffectiveness of screening for HIV in the era of highly active antiretroviral therapy," New England Journal of Medicine, vol. 352 , no. 6, pp. 570-585, 2005.

[3] World Health Organization (WHO), Antiretroviral Therapy (ART), WHO, Geneva, The Switzerland, 2006.

[4] D. Kirschner, "Dynamics of co-infection with M. tuberculosis and HIV-1," Theoretical Population Biology, vol. 55, no. 1, pp. 94109, 1999.

[5] S. M. Moghadas and A. B. Gumel, "An epidemic model for the transmission dynamics of hiv and another pathogen," ANZIAM Journal, vol. 45, no. 2, pp. 181-193, 2003.

[6] B. G. Williams and C. Dye, "Antiretroviral drugs for tuberculosis control in the era of HIV/AIDS," Science, vol. 301, no. 5639, pp. 1535-1537, 2003.

[7] T. C. Porco, P. M. Small, and S. M. Blower, "Amplification dynamics: predicting the effect of HIV on tuberculosis outbreaks," Journal of Acquired Immune Deficiency Syndromes, vol. 28, no. 5, pp. 437-444, 2001.

[8] R. W. West and J. R. Thompson, "Modeling the impact of HIV on the spread of tuberculosis in the United States," Mathematical Biosciences, vol. 143, no. 1, pp. 35-60, 1997.

[9] R. Naresh and A. Tripathi, "Modelling and analysis of HIV-TB co-infection in a variable size P," Mathematical Modelling and Analysis, vol. 10, no. 3, pp. 275-286, 2005.

[10] R. Naresh, D. Sharma, and A. Tripathi, "Modelling the effect of tuberculosis on the spread of HIV infection in a population with density-dependent birth and death rate," Mathematical and Computer Modelling, vol. 50, no. 7-8, pp. 1154-1166, 2009.

[11] E. F. Long, N. K. Vaidya, and M. L. Brandeau, "Controlling Coepidemics: analysis of HIV and tuberculosis infection dynamics," Operations Research, vol. 56, no. 6, pp. 1366-1381, 2008.

[12] M. Martcheva and S. S. Pilyugin, "The role of coinfection in multidisease dynamics," SIAM Journal on Applied Mathematics, vol. 66, no. 3, pp. 843-872, 2006.

[13] G. J. Butler and P. Waltman, "Bifurcation from a limit cycle in a two predator-one prey ecosystem modeled on a chemostat," Journal of Mathematical Biology, vol. 12, no. 3, pp. 295-310, 1981.

[14] P. Magal, C. C. McCluskey, and G. F. Webb, "Lyapunov functional and global asymptotic stability for an infection-age model," Applicable Analysis, vol. 89, no. 7, pp. 1109-1140, 2010.

[15] E. M. C. D’Agata, P. Magal, and S. G. Ruan, "Asymptotic behavior in nosocomial epidemic models with antibiotic resistance," Differential and Integral Equations, vol. 19, no. 5, pp. 573-600, 2006.

[16] M. Iannelli, "Mathematical theory of age-structured population dynamics," in Applied Mathematics Monographs 7, comitato Nazionale per le Scienze Matematiche, Consiglio Nazionale delle Ricerche (C.N.R.), Pisa, Italy, 1995.

[17] H. R. Thieme, "Persistence under relaxed point-dissipativity (with application to an endemic model)," SIAM Journal on Mathematical Analysis, vol. 24, no. 2, pp. 407-435, 1993.

[18] J. K. Hale and P. Waltman, "Persistence in finite dimensional systems," SIAM Journal on Mathematical Analysis, vol. 20, no. 2, pp. 388-395, 1989.

[19] Centers for Disease Control and Prevention (CDC), Coinfection With HIV and Hepatitis C Virus, CDC, Atlanta, Ga, USA, 2006. 


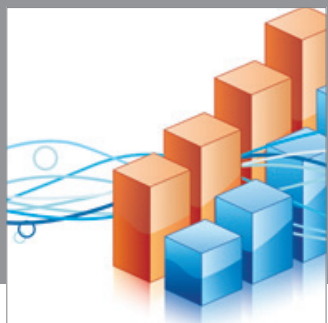

Advances in

Operations Research

mansans

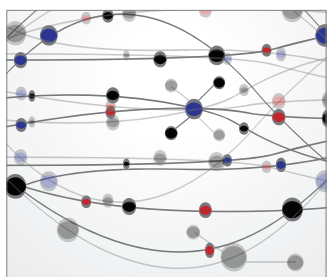

The Scientific World Journal
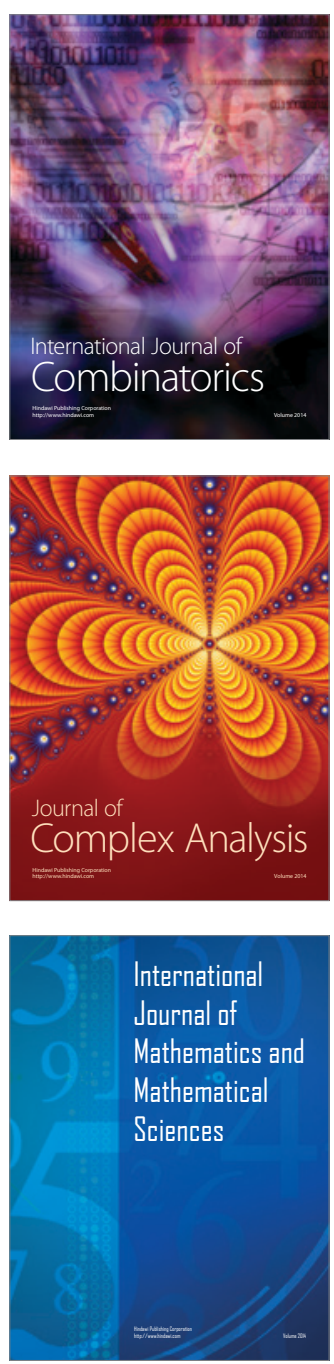
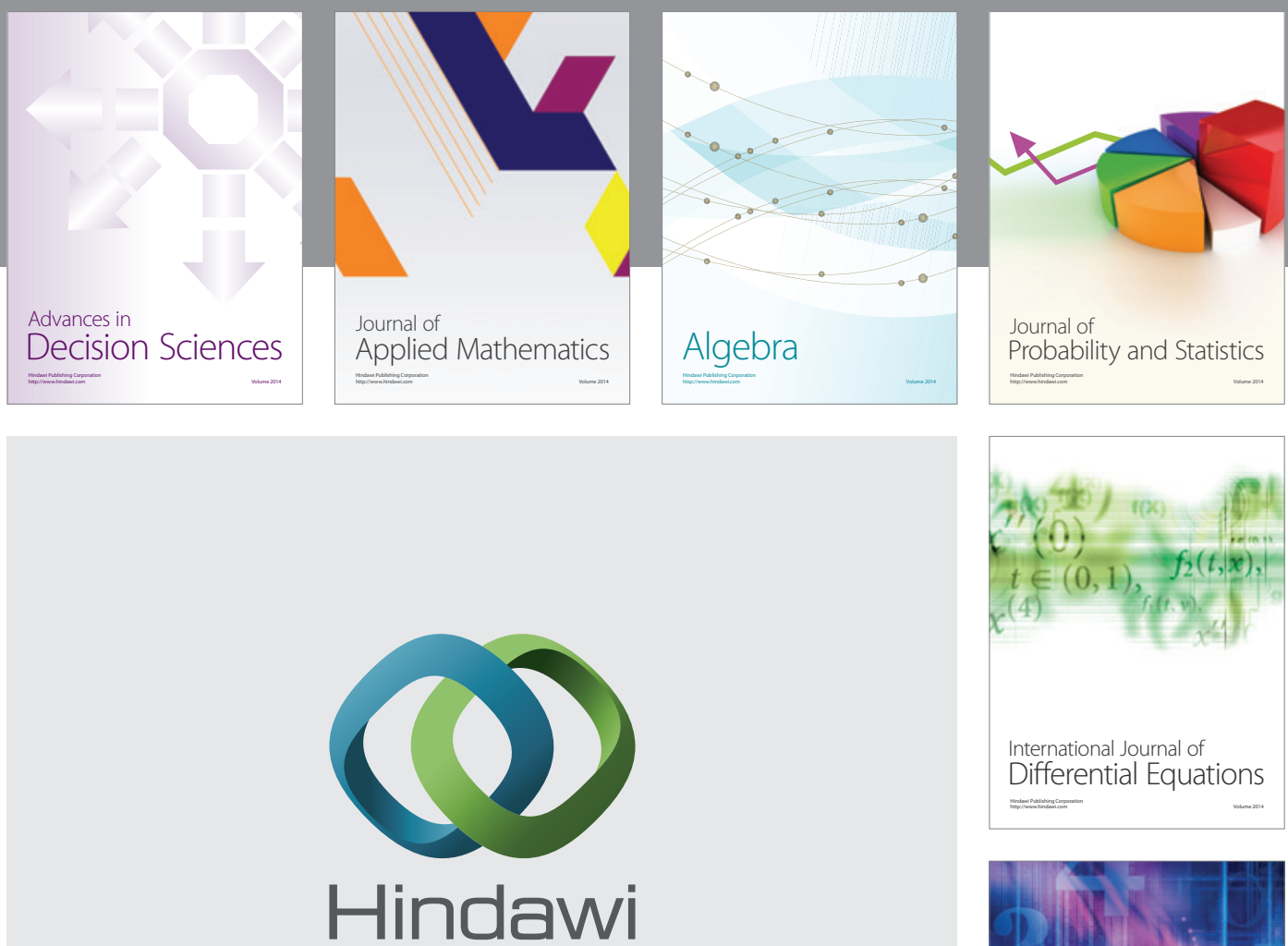

Submit your manuscripts at http://www.hindawi.com
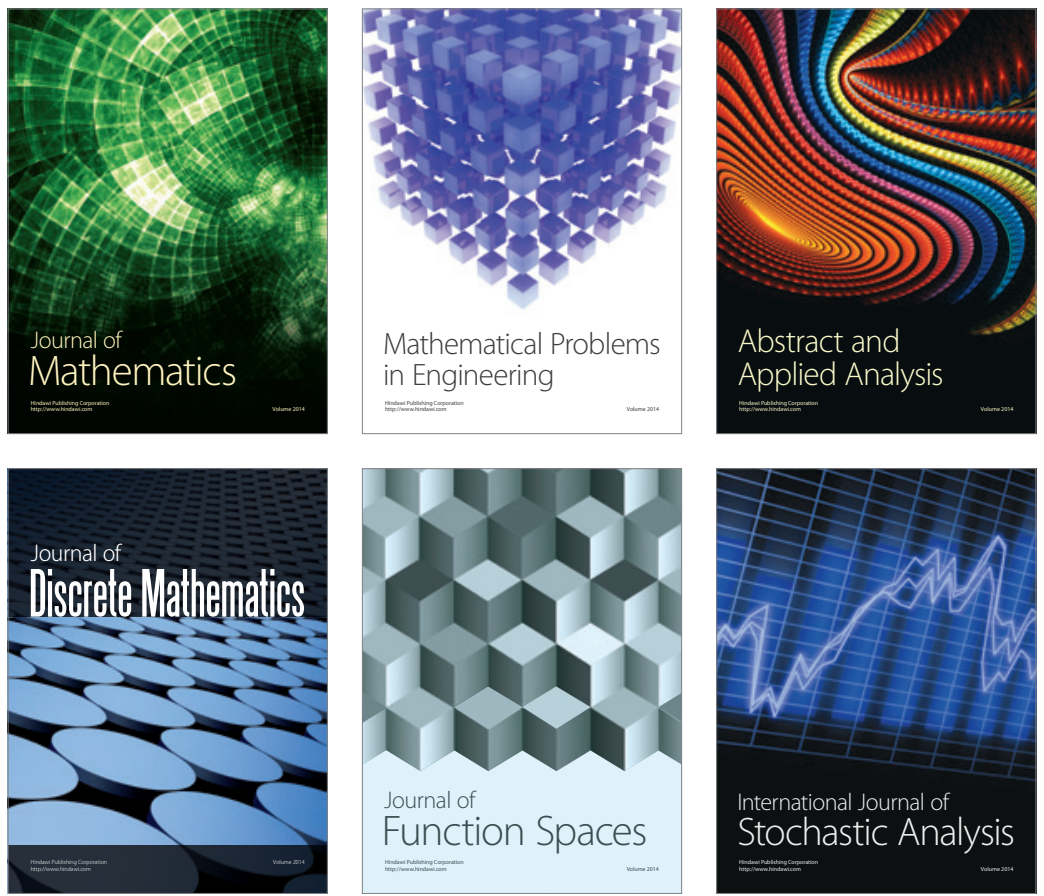

Journal of

Function Spaces

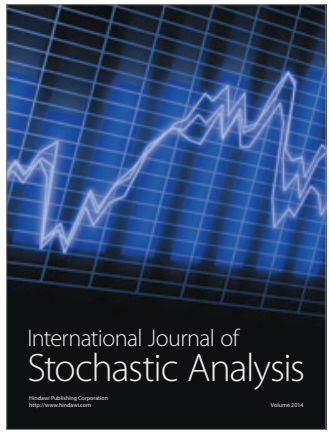

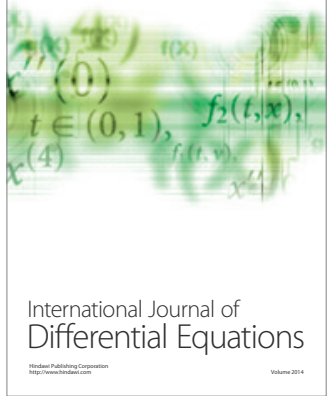
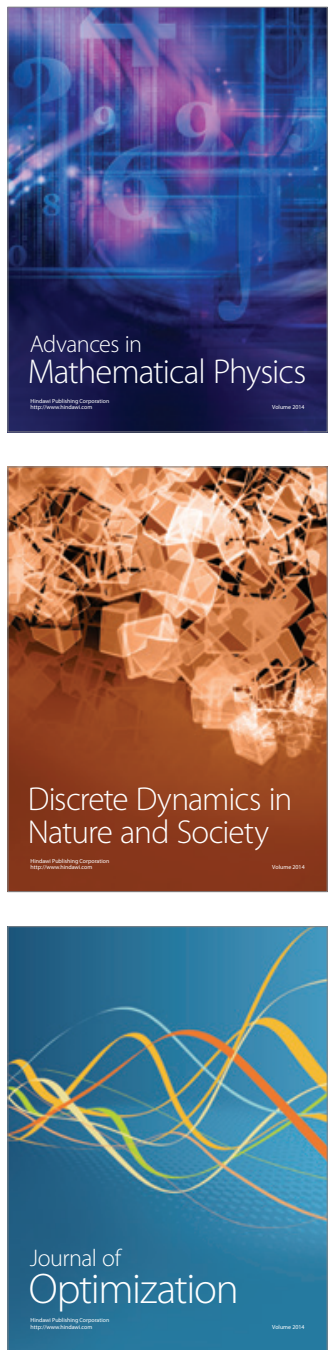